\title{
Surface Plasmon Resonance Biosensor in Healthcare Application Using Kretschsmann \& Otto Configuration
}

\author{
Nusrat Alim and Mohammad Nasir Uddin, Member, IEEE
}

\begin{abstract}
In this study, a planar D-shape optical fiber sensor based on Surface Plasmon Resonance phenomenon using two different excitation methods has been demonstrated. The proposed bio-sensor uses Amplitude-Wavelength hybrid detection technique to determine the change in urine refractive index that depends on resonance parameters. The analysis employs finite element method to investigate the optical properties of the proposed sensor. From the results it can be evident that Otto configuration offers greater amount of reflected light intensity dip for a particular urine refractive index in comparison with Kretschmann configuration. For an example, for Otto configuration the dip of reflected light intensity measured in terms of reflectance which attains a value 0.5 for $\mathrm{ns}$ (urine refractive index) of 1.489 while Kretschmann configuration provides a dip of 0.65 for the same value of $n s$. Results also show that the sharpness of reflectance is comparatively higher for the sensor using Otto configuration in comparison with Kretschmann configuration in case of wavelength interrogation. Thus, Otto configuration offers better performance in comparison with Kretschmann configuration.
\end{abstract}

Keywords - Surface Plasmon Resonance (SPR), optical fiber sensor (OFS), Kretschmann configuration, Otto configuration, Amplitude- Wavelength hybrid detection;

\section{INTRODUCTION}

Acute chronic renal disease, dehydration, nephropathy may cause severe health risk and ultimately lead to renal damage, heart stroke and death. Hence early detection of renal function state and dehydration status are essential for prevention and treatment of aforesaid diseases [1]. For this purpose, specific gravity and osmic pressure of urine can play an important role to identify both renal function state and dehydration status [2]. Both of them can be measured through measuring urine refractive index as there is a correlation that exists between total amount of solids dissolved in the urine sample and its refraction. Urinometer and Refractometer are two commonplace means of measuring solids dissolved in the urine sample and its refraction [3].

Nusrat Alim is a student of Masters in Electrical and Electronic Engineering (MEEE), American International University-Bangladesh, Banani, Dhaka-1213, Bangladesh.

Email:nusratalim@gaim.com

Mohammad Nasir Uddin is an Associate Professor of Department of Electrical and Electronic Engineering, American International UniversityBangladesh, Banani, Dhaka-1213, Bangladesh

Email: drnasir@aiub.edu
Resonance based optical sensing technique can be used for efficient monitoring of molecular binding in real time for applications such as biological and chemical sensing [4]. It is widely used in the field of medical diagnosis, chemical vapor detection, food safety measurement and environmental monitoring due to its superior refractive index sensing property [5]. Sensitivity of SPR biosensor is the fundamental reason to use it for such applications [4]. SPR technology is based on the electromagnetic field component of incident light that penetrates tens of nanometers into the surface of a material [6]. Due to transfer of energy between evanescent wave and surface plasmon, coherent electron oscillation occurs at metaldielectric interface. As a result a stimulated resonance condition occurs at the interface that causes a sharp reduction in reflected light intensity [7]. There are two configurations used to excite surface plasmon wave at the metal-dielectric interface: One is Otto configuration [8] and another one is Kretschmann configuration [9]. Both of the configurations use attenuated total reflection method (ATR) to excite surface plasmons [10]. Kretschmann- Raether configuration is less complex to fabricate compared to Otto configuration. But Kretschmann- Raether configuration offers a fixed dielectric gap sandwiched between two other dielectric mediums that eventually affects the sensitivity of measurement [11]. On the other hand, Otto-configuration involves a bit complex fabrication process but provides better sensitivity than Kretschmann configuration [12]. An improvement in the sensitivity of both type of SPR sensor can be introduced through exploiting gold nanoparticles that functionalizes at the metal- dielectric interface [4]. In this paper, a theoretical study using both of the configurations has been done with the help of numerical approaches to investigate the change in optical properties of light wave dependent on the variation of refractive index of dielectric medium [13]. The proposed optical fiber sensor is suitable for embedding in public toilets. Elderly people can take the advantage out of it as they often have to experience urinal infection and need to go for a regular checkup. Therefore, the proposed device can act as an initial indicator to identify possible health issues through urinalysis.

In this paper, Wavelength- Amplitude Hybrid detection scheme has been followed to establish a fundamental relationship between the resonance parameters and refractive index variation of urine sample (over the range of 1.33001.3489 ) by dint of using a D-shape optical fiber sensor based on SPR technology [14]. Both Kretschmann- Rathether and 
Otto configurations used for Surface Plasmon excitation have been optimized with a metal coating [15]. A metal coating of $\mathrm{Au}$ layer is deposited in between two dielectric mediums in case of Kretschmann configuration. Likewise, an Au layer is placed at the outer layer of SPR sensor for Otto configuration. Real part of dielectric constant of Au layer must change sign along with metal- dielectric interface to give rise to SPR excitation. Chemical stability and linear behavior with the change of the refractive index of sensing medium have made it one of the promising materials to be used while designing a biosensor [16]. Surface plasmon resonance phenomenon occurs only when the wave-vector matches between an incident light wave and plasmonic wave at the metal-dielectric interface at a particular combination of incident angle, phase, wavelength of incident light and dielectric functions of metal and dielectric sensing medium [17-18]. The phenomenon has received enormous popularity in measuring bimolecular interactions in various reagents and binding agents [19].The detection process that uses Surface Plasmon Resonance phenomenon for determining human health parameters is precisely controlled through maintaining a guided flow of urine-sample solutions [20]. Finally, a study has been made to analyze the change in urine refractive index with the variation of incident angle and wavelength of incident light for both Otto and KretschmannRathether configuration [3].

This paper demonstrates the following sections. Firstly, introduction part covers a brief explanation of SPR technique and its usage in determining change in refractive index of sensing medium with the change in resonant parameters. Secondly, design oriented issues of the proposed D-type optical fiber sensor based on SPR technique are incorporated in the design concept and device arena. Thirdly, results and discussions part reveals a comparison between SPR sensors based on Kretschmann and Otto configuration in terms of magnetic field distribution at metal-dielectric interface, amplitude interrogation and wavelength interrogation. Finally, conclusion of the paper provides a brief discussion about the overall outcomes obtained from this work.

\section{Methodology}

In this work, a 2D structure of a SPR based D-shaped optical fiber sensor has been presented considering both Kretschmann and Otto configurations. The Fresnel equations analysis method has been employed in Comsol Multiphysics version 4.4 to investigate both of the two configurations used to excite surface plasmon wave. For this purpose, finite element method (FEM) has been used to study the optical behavior of proposed fiber sensor [21]. The finite element method (FEM) is a comprehensive tool for various physics and engineering applications, especially coupled phenomena, or multi-physics. It is a simulation platform to calculate resonant angles as well. TM (transverse magnetic) or parallel polarized light wave having a wavelength of $632.8 \mathrm{~nm}$ and an incident angle of $67.25^{\circ}$ is used to cause the coherent electron oscillation at metal- dielectric sensing medium and a thin gold film layer with a thickness of $50 \mathrm{~nm}$ has been used for optimal excitation of Surface Plasmon Wave [22]. The resonance condition is established when the frequency of light photons matches the natural frequency of surface electrons oscillating against the restoring force of positive nuclei. Wavelength of incident light, incident angle, metal layer properties and geometry of the sensing device have a great influence on the coupling of evanescent wave with surface plasmon wave. For the terms which describe the electronic surface plasmon to exist, the real part of the dielectric constant of the metal must be negative and its magnitude must be greater than that of the dielectric. In this phenomenon, smaller values of incident angle are responsible for causing greater number of reflections per unit length of the device [23]. Resonance condition depends on the type of material used as a sensing medium that further allows characterization of SPR phenomenon.

\section{DEVICE CONCEPT AND DESIGN}

The paper mainly investigates the Amplitude-Wavelength hybrid detection technique in order to detect the change in urine refractive index in accordance with the change of incident angle and light wavelength. In Fig. 1. a planar waveguide of proposed optical sensor has been shown with core dielectric constant 2.28, cladding dielectric constant 2.12, metal dielectric constant $-25+1.44 \mathrm{i}$ [10] and metal thickness $50 \mathrm{~nm}$, urine refractive index ranges from 1.3374-1.3489. Through symmetrical removal of a portion of cladding, the proposed SPR technology based D-type optical sensor has been designed.

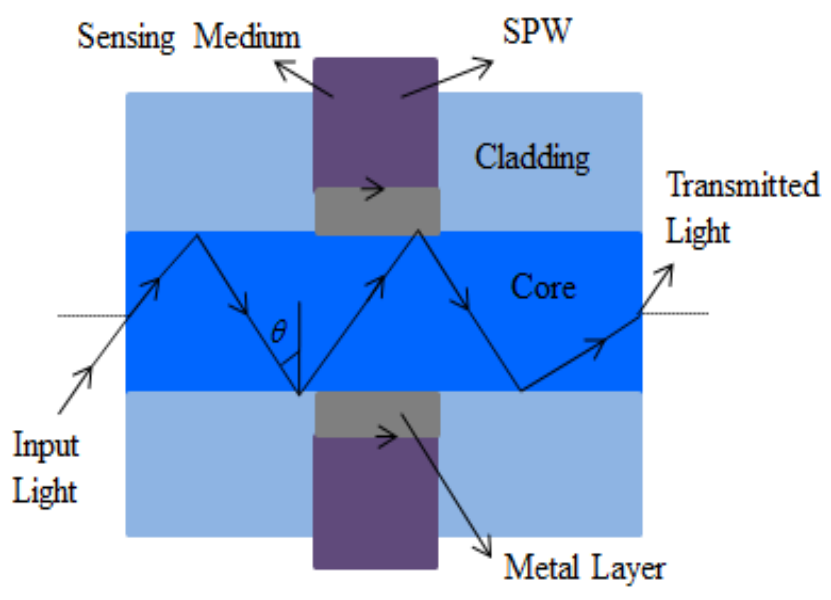

Fig. 1. Schematic Diagram of D-shape fiber optic sensor based on surface plasmon resonance using Kretschmann configuration

In the above schematic diagram a planar waveguide has been designed using Kretschmann configuration where a metallic layer (gold) has been sandwiched in between two dielectric medium [24]. In similar fashion, Otto configuration can be designed by interchanging the position of metal and sensing dielectric medium.

\section{RESULTS AND DISCUSSIONS}

Urine samples having different refractive indices are considered as sensing medium. Reflectance profile of the proposed sensor for both Kretschmann- Rathether and Otto configurations have been investigated by dint of Amplitude and Wavelength interrogation. Results obtained from simulation work reveals the full potential of SPR biosensor: when wavelength shifts and amplitude of reflected light wave varies in accordance with the variation of sensing medium refractive index. Refractive index of urine sample ranges between 1.3374-1.3489 [2], pointing as a possible indicator for determining health related problems. The resonant angle of the proposed sensor computed here is $67.25^{\circ}$ that complies with both of the configurations. In this paper a comparison between two of the configurations has been made through investigating magnetic field distribution, reflectance profile through varying 
angle of incidence, wavelength of light. The results confirms its suitable usage as a diagnosis tool of urinalysis to detect possible health issues.

\section{A. Magnetic field distribution at the surface of SPR:}

The distribution of magnetic field in the aforesaid structures at resonant angle are shown in Fig 2(a) and Fig. 2(b) below. At resonant angle for Kretschmann- Rathether configuration magnetic field intensity experiences an exponential decay at the gold-sensing dielectric interface [25]. On the other hand, an exponential decay is encountered at prism - sensing dielectric medium interface. For same design parameters Otto configuration offers slightly better magnetic field intensity compared to Kretschmann configuration. For wavelength of $632.8 \mathrm{~nm}$ the respective peak values of magnetic field intensity for both Otto and KretschmannRathether configuration are respectively given as $1218 \times 10^{3}$ $\mathrm{A} / \mathrm{m}$ and $1215 \times 10^{3} \mathrm{~A} / \mathrm{m}$.

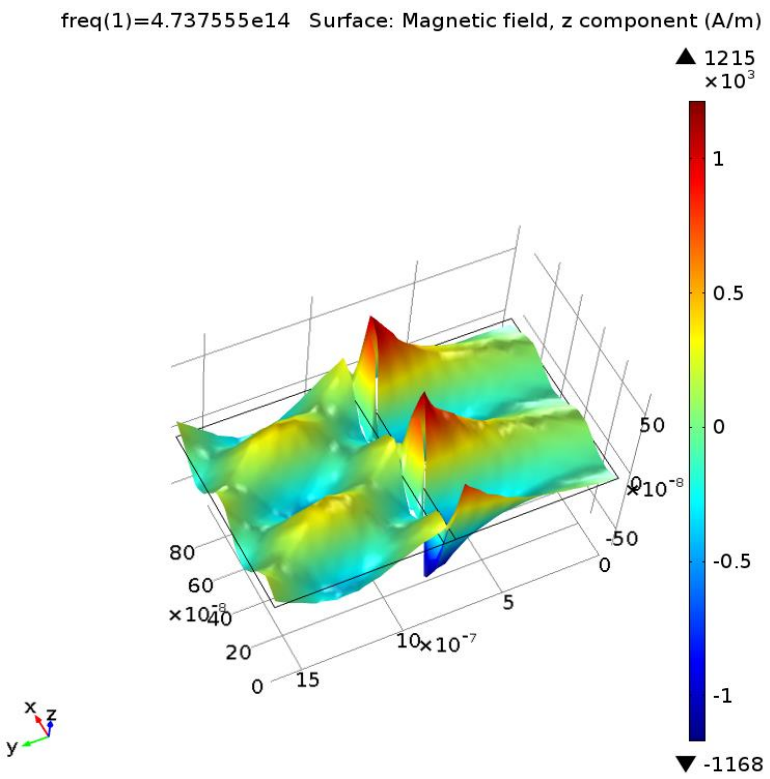

Fig. 2. (a). Magnetic field distribution at resonance condition for Kretschmann-Raether configuration.

In this paper, $\mathrm{z}$-component of magnetic field $(\mathrm{A} / \mathrm{m})$ has been shown for both of the SPR configurations. Classical eletromagnetic field theory considering eleltromagnetic wave reflection, transmission and absorption for materials consisted of multiple layers can be used to get a close insight into SPR phenomenon [6]. Surface plasmon resonance formed at resonance condition propagates along the metal-dielectric interface and the magnectic field distribution has a great influence to depict the surface plasmon resonance effect on electromagnetic field. Thus it is essential for characterization of surface associated phenomenons required to investigate biomolecular interactions between reagents and analytes. Commercial deployment of SPR technology for measuring surface contaminents at a nano-scale range has witnessed a major breakthrough in the development of medical science.

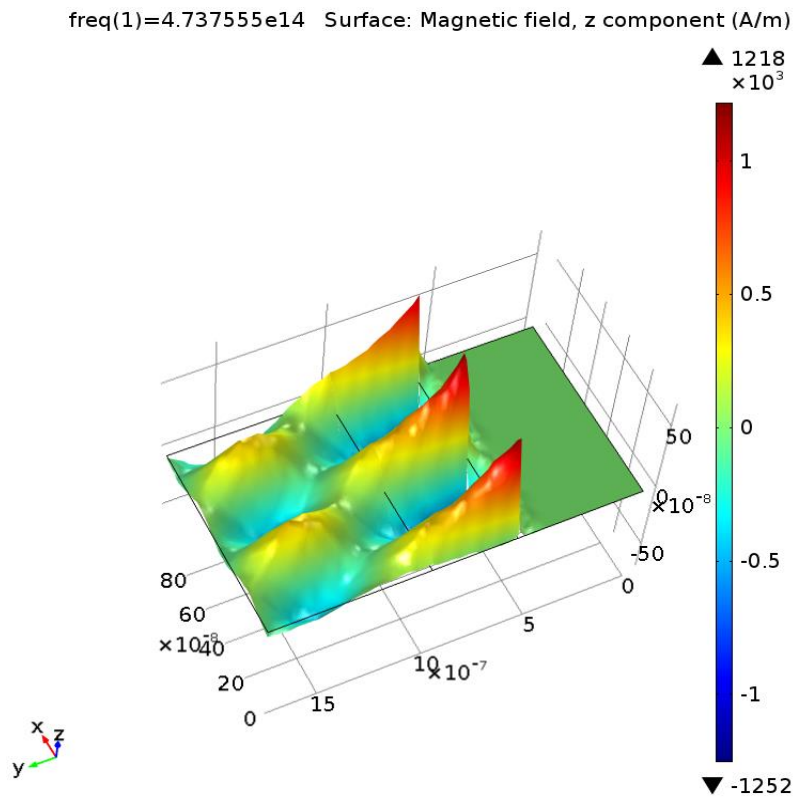

Fig. 2. (b). Magnetic field distribution at resonance condition for Otto configuration.

\section{B. Amplitude Detection:}

Fig. 3 (a) and Fig. 3(b) show a sharp decline in the reflected light intensity for higher values of refractive index of urine sample at a resonant incident angle of $67.25^{\circ}$ for both of the configurations at a wavelength of $632.8 \mathrm{~nm}$. A few samples of urine reflective indices are taken to identify "healthy group, "sick-group" and "treated group" [14]. For Kretschmann configuration the dip of reflected light intensity drops from 0.92 to 0.65 as the refractive index approaches towards higher values ranges from 1.3374 to 1.3489 . In case of Otto configuration the dip in reflected light intensity declines from 0.90 to 0.50 when there is an increase in urine refractive index holding values of 1.3374 to 1.3489 .

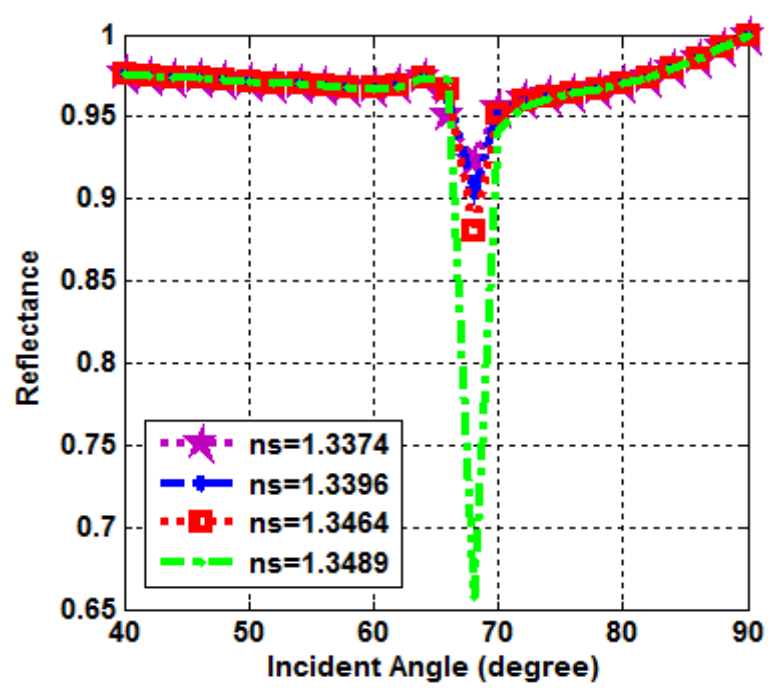

Fig. 3. (a). Reflectance as a function of incident angle for Kretschmann configuration.

Therefore, these results state a relationship between urine refractive index and incident angle which dramatically affects the optical properties of the proposed sensor [24-25]. Thus, a change in reflected light intensity can be used as a measure of change in refractive index of sensing medium. Eventually, the 
value of urine specific gravity (USG) can be determined through knowing the urine refractive index (RI).

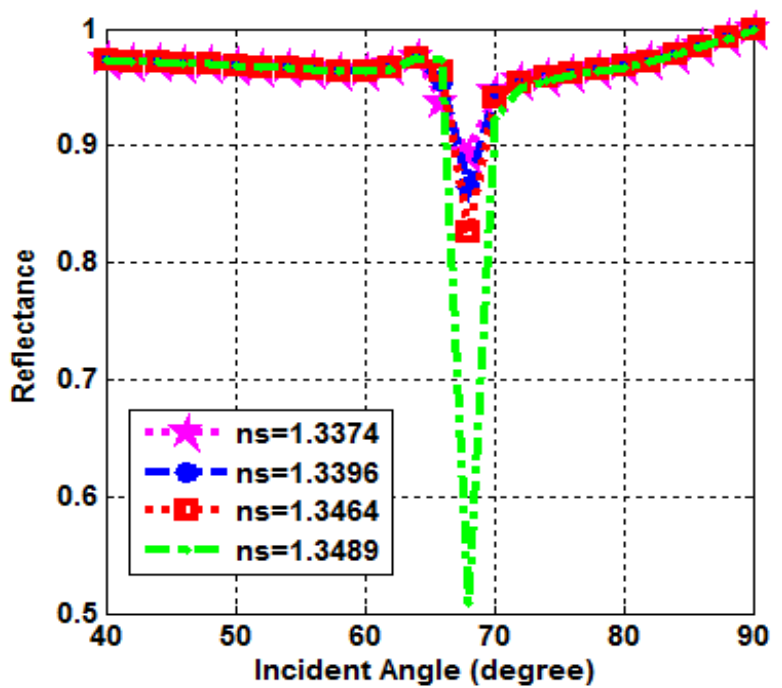

Fig. 3. (b). Reflectance as a function of incident angle for Otto configuration.

\section{Wavelength Detection:}

In case of Wavelength detection the wavelength of incident light wave is varied in between $200 \mathrm{~nm}$ to $1600 \mathrm{~nm}$ that covers the wavelengths fall into visible spectrum $(400 \mathrm{~nm}$ to $700 \mathrm{~nm}$ ) and infrared region. A wavelength shift heading towards smaller wavelengths can be observed when the refractive index of the sensing medium increases from 1.3374 to 1.389 , applicable for both of the configurations.

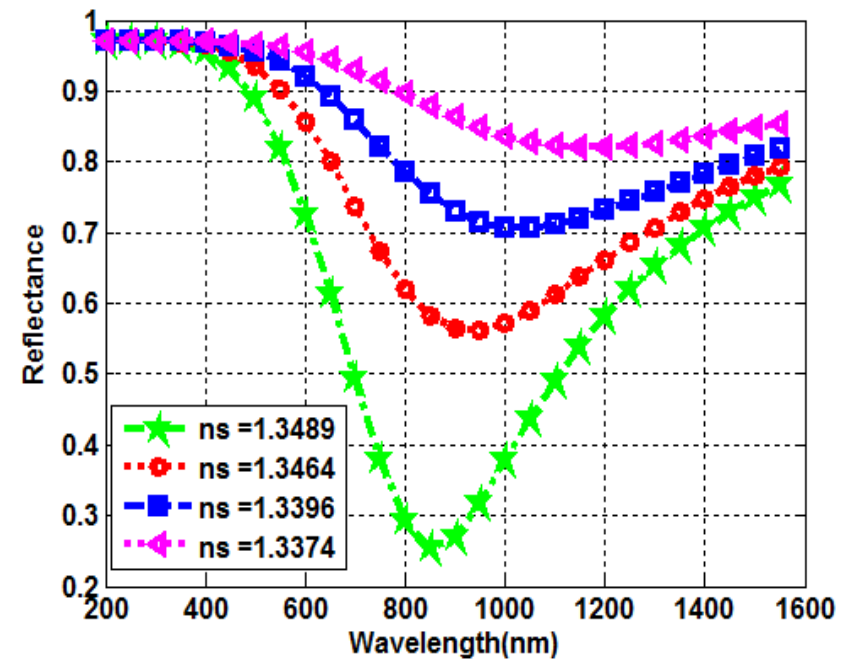

Fig. 4. (a). Surface Plasmon Resonance spectra at different values of urine refractive indices for Kretschmann- Rathether configuration.

From Fig. 4(a) and Fig. 4(b) it can also be evident that for Otto configuration at $67.25^{\circ}$ incident angle FWHM (full width at half maximum) values of incident wavelength attain comparatively smaller values in comparison with FWHM values obtained from Kretschmann- Rathether configuration for a change in urine refractive index. For example, in case of Kretschmann- Rathether configuration approximately $550 \mathrm{~nm}$ FWHM value can be figured out at a urine refractive index of 1.3489. Likewise, Otto configuration provides a value of 350 $\mathrm{nm}$ for urine refractive index of 1.3489. Hence, the following figures depict that sharper curves provide higher sensitivity. In this regard, sensor designed using Otto configuration offers better sensitivity compared to Kretschmann- Rathether configuration as because the narrower the line width the higher the sensitivity. For a specific value Au thickness the measurement sensitivity of the proposed sensor can be further improved to a great extent.

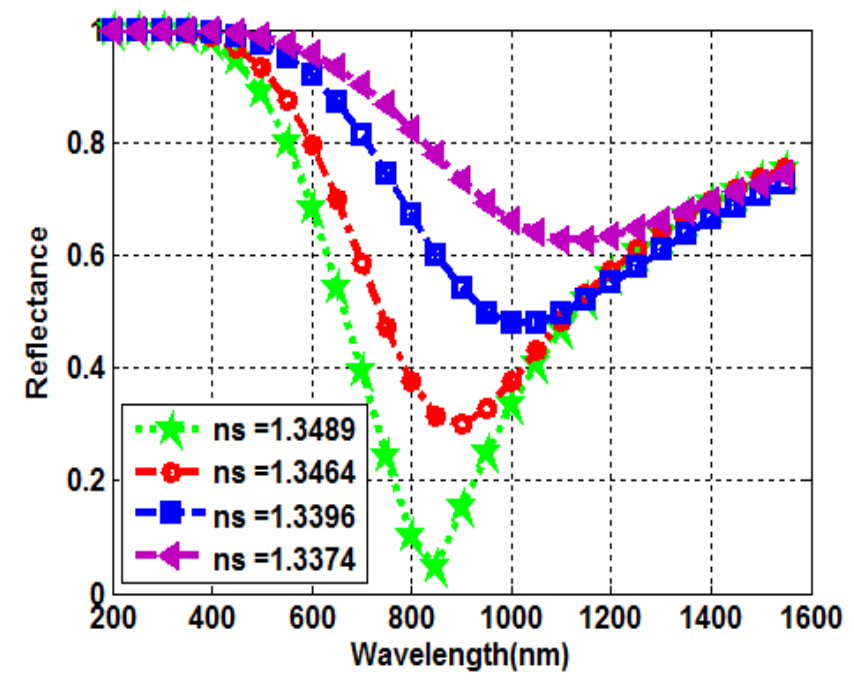

Fig. 4. (b). Surface Plasmon Resonance spectra at different values of urine refractive indices for Otto configuration.

\section{CONCLUSION}

The simulation work presents a SPR based optical sensor with two different configurations of surface plasmon wave excitation. From the results, it can be observed that the urine refractive index has a great influence on resonance angle and resonance wavelength. With the increase in urine refractive index the dip of reflected light intensity falls from 0.92 to 0.65 for Kretschmann- Rathether configuration. Whereas Otto configuration exhibits a decrease in the reflectance dip holding values from 0.90 to 0.5 for an increase in refractive index of urine sample. Both of the results are obtained for amplitude interrogation. Likewise, in case of wavelength interrogation resonant wavelength of incident light wave attains shorter wavelengths as the refractive index of urine sample approaches toward higher values. In this work, Otto configuration exhibits smaller full width half maxima values for a particular refractive index compared to Kretschmann configuration. Thus, urine refractive index can be detected through amplitude measurement and wavelength interrogation using both of the configurations. It can also be said that Otto configuration provides higher dips in reflected light intensity as well as narrower FWHM values with respect to urine refractive index variation compared to Kretschmann configuration though Kretschmann is comparatively easy to fabricate than Otto configuration. In our future work, we would like to fabricate the proposed optical sensor by means of implementing both of the configurations.

\section{ACKNOWLEDGMENT}

The work is partially supported by the IEEE Educational Activity Coordinator (EAC), Bangladesh Section. 


\section{REFERENCES}

[1] T. Guo, F. Liu, X. Liang, X. Qiu, Y. Huang, C. Xie, P. Xu, W. Mao, B $\mathrm{O}$. Guan and J. Albert, Highly sensitive detection of urinary protein variations using tilted fiber grating sensors with plasmonic nanocoatings, Biosensors and Bioelectronics, vol. 78, April. 2016, pp. 221-228.

[2] A.V. Wolf, Aqueous solutions and body fluids, their concentrative properties and conversion tables, Hoeber Medical division, Harper \& Row, New York, 1966.

[3] L. Bo, P. Wang and Y. Semenova, High Sensitivity Fiber Refractometer Based on an Optical Microfiber Coupler, IEEE Photonics Technol. Lett., vol. 25, issue 3, Feb. 2013.

[4] M. Kashif, A. A. A. Bakar, N. Arsad and S. Shaari, Development of Phase Detection Schemes Based on Surface Plasmon Resonance Using Interferometry, Sensors, vol. 14, Aug. 2014, pp. 15914-15938.

[5] T. Hu, Y. Zhao, A. Song, Fiber optic SPR sensor for refractive index and temperature measurement based on MMF-FBG- MMF structure, Sensor and Actuators B, vol. 237, Jun. 2016, pp. 521-525.

[6] J. Crompton, S. Yushanov, L. T. Gritter and K. C. Koppenhoefer, Analysis of Surface Plasmon Resonance, COMSOL Conference, Boston, USA, 2012.

[7] N. Cennamo, D. Massarotti, R. Galatus, L. Conte and L. Zeni, Performance Comparison of Two Sensors Based on Surface Plasmon Resonance in a Plastic Optical Fiber, Sensors, vol. 13, Jan. 2013, pp. 721-735,

[8] J. O. M. Neto , G. O. Cavalcanti , I. L. Garro , J. M. Kim and E. Fontana, Pressure sensing by surface plasmon resonance in the otto configuration, IEEE Sensors, Jan. 2017 pp. 1722-1723.

[9] C. Zhao, Y. Wang, D. Wang and Z. Ding, Numerical Investigation into a Surface Plasmon Resonance Sensor Based on Optical Fiber Microring, Photonic Sensors, vol. 7, no. 2, Nov. 2016, pp. 105-112.

[10] O. S. Wolfbeis and J. Homola, Fundamentals of SPR Sensors, Springer Ser Chem Sens Biosens, Berlin, Heidelberg, vol. 4, Jul. 2006,pp. 3-44.

[11] N. Chiu, Y. C. Tu and T. Y. Huang, Enhanced Sensitivity of Antisymmetrical Structured Surface Plasmon Resonance Sensors with Zinc Oxide Intermediate Layer, Sensors, vol. 4, Dec. 2014, pp. 170-187.

[12] Ermini, M. L. Scarano and S. Minunni, Surface nanostructuring for Surface Plasmon Resonance imaging, International Workshop on BioPhotonics, Parma, Italy, 8-10 Jun 2011, pp. 1-3.

[13] L. Ji, Y. Chen and Y. J. Yuan, Investigation of surface plasmon resonance phenomenon by finite element analysis and Fresnel Calculation, Sensors and Actuators B: Chemical, vol. 198, Mar. 2014, pp. 82-86.

[14] T. Guo, F. Liu, X. Liang, X. Qiu, Y. Huang, C. Xie, P. Xu, W. Mao, B. O. Guan and J. Albert, Highly sensitive detection of urinary protein variations using tilted fiber grating sensors with plasmonic nanocoatings, Biosensors and Bioelectronics, vol. 78, April. 2016, pp. 221-228.

[15] J. Albert, S. Lepinay, C. Caucheteur and M. C. DeRose, High resolution grating-assisted surface plasmon resonance fiber optic aptasensor, Methods, vol. 63, July 2013, pp. 239-254.

[16] N. K. Sharma, M. Rani, V. Sajal, Surface plasmon resonance based fiber optic sensor with double resonance dips, Sensors and Actuators B: Chemical, vol. 188, July 2013, pp. 326-333.

[17] S. Chen and C. Lin, High-performance bimetallic film surface plasmon resonance sensor based on film thickness optimization, Optik, vol. 127, pp. 7514-7519, May. 2016.

[18] S. P. Yushanov, L. T. Gitter, J. S. Crompton and K. C. Koppenhoefer, Surface Plasmon Resonance, COMSOL Conference, USA, Oct. 2012.

[19] M. Li, S. K. Cushing and N. Q. Wu, Plasmon-enhanced optical sensors: a review, Analyst, vol. 140, no. 2, Jan. 2015, pp. 386-406.

[20] D. F. SANTOS, A. GUERREIRO and J. M. BAPTISTA, Numerical Investigation of a Refractive Index SPR D-Type Optical Fiber Sensor Using COMSOL Multiphysics, Photonic Sensors, vol. 3, no. 1, Jul. 2013, pp. $61-66$,
[21] M. Yang, K. Xia, X. Feng, Y. Luo, J.Tang, J. Fang, J. Zhang, H. Lu, J. $\mathrm{Yu}, \mathrm{Y}$. Chen and Z. Chen, Design and Optimization of Surface Plasmon Resonance Fiber Sensor Based on Gold Nano-Column Array, NUSOD International Conference, Australia, Aug. 2016.

[22] M. H. Chiu, Ch. H. Shih and M. H. Chi, Optimum sensitivity of singlemode D-type optical fiber sensor in the intensity measurement, Sensors and Actuators B, vol. 123, pp. 1120-1124, Jan. 2007.

[23] A. K Sharma, R. Jha and B. D. Gupta, Fiber-optic sensors based on surface plasmon resonance: a comprehensive review, IEEE Sensors J., vol. 7, no. 8, Aug. 2007, pp. 1118-1129.

[24] N. Alim, M. N. Uddin, Surface Plasmon Resonance in Healthcare Application, IEEE TENSYMP 2017, Cochin, Kerala, India, July 2017 [accepted].

[25] N. Chiu, Y. C. Tu and T. Y. Huang, Enhanced Sensitivity of Antisymmetrical Structured Surface Plasmon Resonance Sensors with Zinc Oxide Intermediate Layer, Sensors, vol. 4, , Dec. 2014, pp. 170-187.

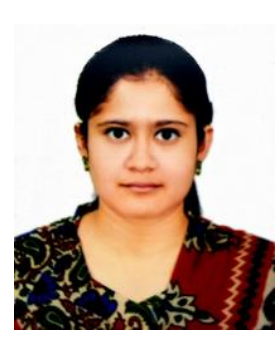

Nusrat Alim received B.Sc. degree in Electrical \& Electronic Engineering from American International University-Bangladesh on February 2014 and now continuing M.Sc. in Electrical \& Electronic Engineering in AIUB. She started her teaching career as a Lecturer of Electrical \& Electronic Engineering Department in Green University of Bangladesh since June 2014. She has also been involved in various quality improvement activities of her respective department at the very outset of her teaching career. She received academic scholarship for her brilliant result in B.Sc.Program. She also received prestigious 'Summa Cum Laude' distinction for her excellent academic record in undergraduate program. Recently, she is continuing her M.Sc. thesis under the conversant supervision of Mohammad Nasir Uddin, Associate Professor, AIUB and her research interest mainly focuses on Optical fiber sensor design, Optical Network design based on DWDM-OFDM-PON. Nusrat Alim is a member of the Institute of Electrical and Electronics Engineers (IEEE).

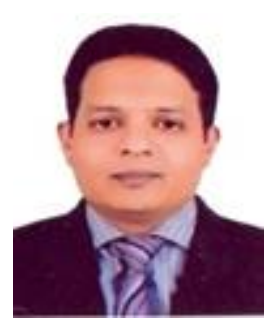

Mohammad Nasir Uddin received B.Sc. degree in Electrical and Electronic Engineering from Khulna University of Engineering and Technology, KUET in 2003 and M.Sc. Engineering in Computer Networks from Middlesex University, United Kingdom (UK) in 2006 and Ph.D. degree from Kyushu University, Japan in 2015. He started his teaching career as a Lecturer of Computer Science \& Engineering Department of University of Development Alternative (UODA), Dhaka, Bangladesh on February 2004. On September 2006, he joined Department of Computing, The Business School of London, United Kingdom (UK) as a Lecturer. He was appointed as a Lecturer of Electrical and Electronic Engineering Department, Faculty of Engineering, American International University-Bangladesh (AIUB) on January 2009 and promoted to Assistant Professor during 2010. Currently he is serving as an Associate professor at the same dept. He received monbukagakusho scholarship from Japan Govt. to peruse his $\mathrm{PhD}$ at Kyushu University, Opto-electronics (Hamamoto) Laboratory, Japan. He has received numerous award including Merit Award during M.Sc at Middlesex University, UK, 2013 IEEE Excellent student award from Japan, Deans Plaque for his outstanding research during $\mathrm{Ph} . \mathrm{D}$. His current research interest includes Active MMI Laser Diode, High Speed Optical Communication, Wireless Communication and Optical Sensor network. Dr. Uddin is a member of the Institute of Electrical and Electronics Engineers (IEEE), a member of the Institute of Electronics, Information, and Communication Engineers (IEICE), Japan and LIFE FELLOW of Institute of Engineers, Bangladesh (IEB). During 2016 he served as Vice Chair of IEEE YP and currently he is serving as an Executive Committee member (EAC) of IEEE Bangladesh Section. 
